\title{
Design and Application of Magnetic-Based Theranostic Nanoparticle Systems
}

\author{
Aniket S. Wadajkar, ${ }^{\mathrm{a}, \mathrm{b}}$, Jyothi U. Menon, ${ }^{\mathrm{a}, \mathrm{b}}$, Tejaswi Kadapure ${ }^{\mathrm{a}, \mathrm{b}}$, Richard T. Tran ${ }^{\mathrm{c}}$, Jian Yang ${ }^{\mathrm{c}, *}$ and \\ Kytai T. Nguyen ${ }^{a, b,{ }^{*}}$
}

${ }^{a}$ Department of Bioengineering, The University of Texas at Arlington, Arlington, TX 76019; ${ }^{b}$ Joint Biomedical Engineer-
ing Program between The University of Texas at Arlington and The University of Texas Southwestern Medical Center,
Dallas, TX 75390, ${ }^{c}$ Department of Bioengineering, The Pennsylvania State University, University Park, PA 16802

Received: February 16, 2012 Revised: May 11, 2012 Accepted: October 21, 2012

\begin{abstract}
Recently, magnetic-based theranostic nanoparticle (MBTN) systems have been studied, researched, and applied extensively to detect and treat various diseases including cancer. Theranostic nanoparticles are advantageous in that the diagnosis and treatment of a disease can be performed in a single setting using combinational strategies of targeting, imaging, and/or therapy. Of these theranostic strategies, magnetic-based systems containing magnetic nanoparticles (MNPs) have gained popularity because of their unique ability to be used in magnetic resonance imaging, magnetic targeting, hyperthermia, and controlled drug release. To increase their effectiveness, MNPs have been decorated with a wide variety of materials to improve their biocompatibility, carry therapeutic payloads, encapsulate/bind imaging agents, and provide functional groups for conjugation of biomolecules that provide receptor-mediated targeting of the disease. This review summarizes recent patents involving various polymer coatings, imaging agents, therapeutic agents, targeting mechanisms, and applications along with the major requirements and challenges faced in using MBTN for disease management.
\end{abstract}

Keywords: Magnetic nanoparticles, polymeric shell, imaging agents, theranostics, therapeutic agents, hyperthermia.

\section{INTRODUCTION}

Theranostic nanoparticles that simultaneously deliver both imaging and therapeutic agents have gained significant attention for disease management in recent years. Disease management not only includes the highly specific diagnosis and treatment of the diseased cells, but also the monitoring of the drug delivery process and therapeutic efficacy [1]. Conventional nanoparticle systems have been previously used to achieve each aspect of disease management separately; however, multiple administrations may be required to fulfill all the necessary functions, which bring concerns of patient compliance and safety [2]. To overcome these limitations, theranostic nanoparticle systems that can perform all the aspects of disease management in a single setting have been developed over the last decade. In particular, magneticbased theranostic nanoparticles (MBTN) are of great interest in disease management due to the numerous advantages these materials possess when in the presence of a magnetic field, and is summarized in Fig. (1). Magnetic nanoparticles (MNPs) are multifunctional agents that can be used: (a) for site-specific magnetic targeting [3], (b) as negative contrast agents in magnetic resonance imaging (MRI) [4], (c) for hyperthermia treatment under alternating magnetic fields [5],

\footnotetext{
*Address Correspondence to these authors at the Department of Bioengineering, The University of Texas at Arlington, 500 UTA Blvd., Arlington, TX 76019, USA, Tel/Fax: 817-272-2540; E-mail: knguyen@uta.edu; or Dr. Jian Yang, Department of Bioengineering, The Pennsylvania State University, W340 Millennium Science Complex, University Park, PA 16802, USA; Tel/Fax: 814-865-1278; E-mail: jxy30@psu.edu
}

and (d) in magnetic field-dependent controlled drug delivery applications [3] collectively, rendering MNPs as ideal candidates in the development of advanced theranostic systems.

MNPs are composed of ferromagnetic elements such as iron, cobalt, nickel, or their oxides and alloys [6]. MNPs made of iron oxide (magnetite $\mathrm{Fe}_{3} \mathrm{O}_{4}$ or magnemite $\mathrm{Fe}_{2} \mathrm{O}_{3}$ ) and gadolinium (chelated organic gadolinium complexes) [7] have been widely used as contrast agents in MRI for biological applications due to their ability to dissociate into iron and oxygen inside the body, which can safely be eliminated and utilized in metabolic and oxygen transport systems [8, 9]. When fabricated into nanoparticles of approximately $10 \mathrm{~nm}$ in diameter, iron oxide nanoparticles begin to exhibit a superparamagnetic behavior (superparamagnetic iron oxide nanoparticles, SPIONs) leading to improved dispersive properties in the absence of a magnetic field, and later guided to accumulate to the site of interest in the presence of a magnetic field, which is of great importance in targeted drug delivery applications [4]. MNPs also possess low cytotoxicity and have been approved by the United States Food and Drug Administration (FDA) for clinical MRI applications $[9,10]$. Numerous studies have explored the potential of MNPs as therapeutic and diagnostic agents for the management of diseases such as cancers and cardiovascular diseases. The following sections briefly describe the coating materials, targeting mechanisms, imaging agents, and therapeutic agents used in MBTN, along with applications and design considerations of MBTN. 


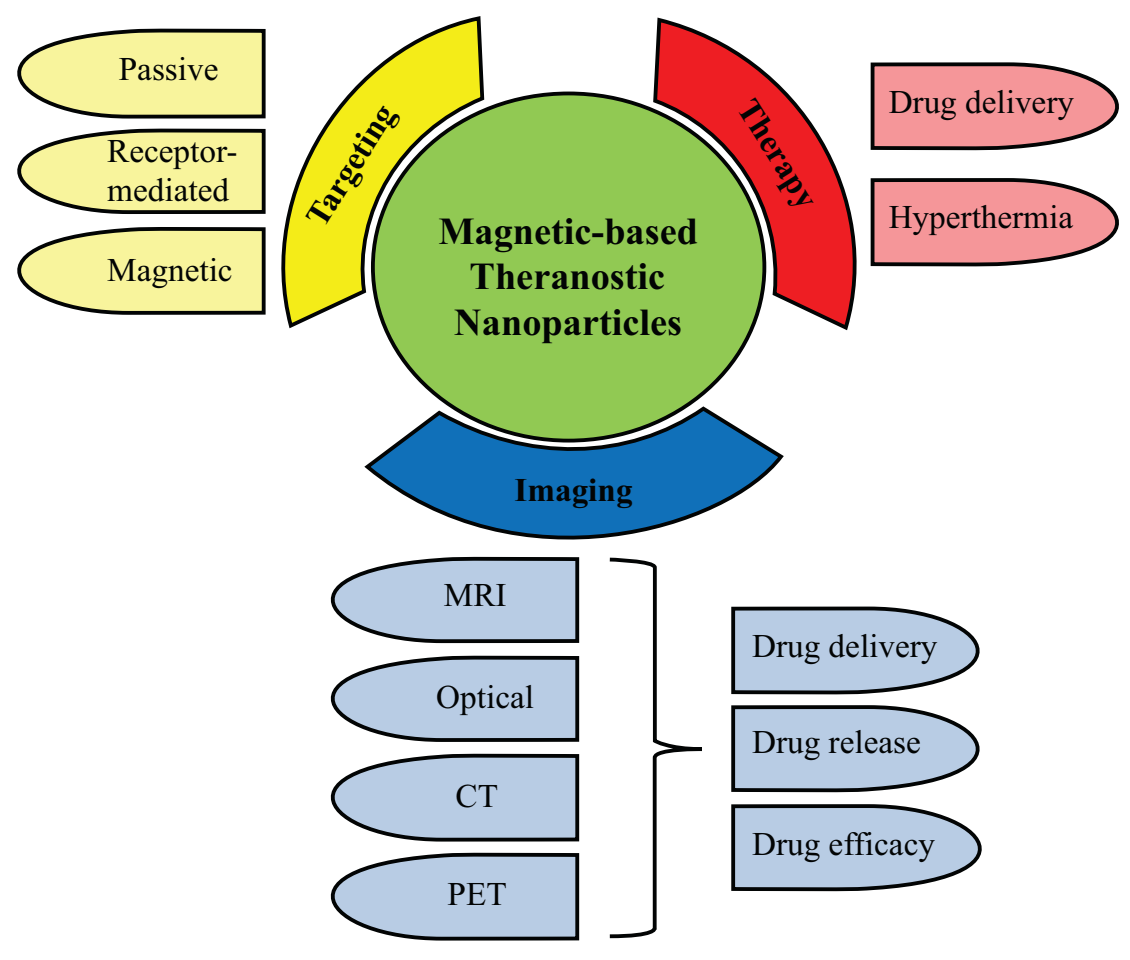

Fig. (1). Attributes and applications of MBTN.

\section{POLYMER COATINGS}

Recent research has been intensely focused on finding suitable biodegradable and biocompatible polymers that can efficiently incorporate drug molecules or imaging agents for delivery, and be decorated with ligands for active targeting of the diseased tissue [7, 11-13]. Hydrophilic natural and synthetic polymers have been used as coating materials due to their ability to prevent particle aggregation, increase solubility, and improve the stability of the particles [1]. Natural polymers such as dextran are gaining prominence in the field since MNPs coated with these polymers have shown improved biocompatibility and tend to stay in circulation for relatively longer periods of time [5]. FDA approved dextrancoated MNPs have already been used to image spleen, liver, and lymph nodes [1]. For instance, dextran-coated MNPs prepared by Tassa et al. [14] imparted both stability and additional functional groups for bioconjugation on the nanoparticle surface. The dextran coating also supported diagnostic imaging of the nanoparticles by MR, optical, and positron emission tomography (PET) imaging. In addition to dextran, chitosan is another common natural material gaining importance as a suitable coating for MNPs due to material's biocompatibility and the added functional groups, which can be utilized for bioconjugation [11]. Microencapsulated MNPs coated with chitosan were injected into the blood vessel leading to the kidney of a New Zealand white rabbit via an angiographic catheter in vivo, and they appeared to be detected in MRI of the kidney $[15,16]$.

In addition to natural materials, MBTN have also been coated with synthetic biodegradable or non-degradable polymers including poly (lactic-co-glycolic acid) (PLGA) [17], poly(glyceryl monooleate) (PGMO) [18], and poly $(N-$ isopropylacrylamide) (PNIPAAm) [12]. PLGA has been widely chosen to coat MNPs by many research groups due to its biocompatible nature and ability to provide the sustained release of encapsulated drugs and/or contrast agents throughout the polymer degradation time to ensure prolong treatment. For example, PLGA-magnetite particles prepared by Chattopadhyay et al. [17] showed sustained drug release for a period of nine hours and could also be used for MRI. Coating of iron oxide nanoparticles using a long chained PGMO as patented by Sahoo et al. [18] improved the aqueous stability of the particles without the use of surfactants. These nanoparticles were loaded with rapamycin $(7.3 \%$ loading) or paclitaxel (7.5\% loading) drugs that showed sustained release kinetics (100\% rapamycin and $80 \%$ paclitaxel was released) over three weeks period. The released of drugs were therapeutically active and showed anti-proliferative effects on breast cancer cells in vitro.

Stimuli-responsive or 'smart' polymers, such as PNIPAAm, have also been used due to their thermo-responsive nature, which enables drug release when the temperature of solution is raised above the lower critical solution temperature (LCST) of the polymers. PNIPAAm copolymerized with acrylamide $(\mathrm{AAm})$ and allylamine $(\mathrm{AH})$ were developed in our laboratory and patented for their use to decorate MNPs $[12,19]$. The PNIPAAm-AAm-AH decorated MNPs shrink and release the encapsulated drugs in response to increase in the surrounding temperature $\left(\sim 39^{\circ} \mathrm{C}\right.$ or above). Combinations of smart polymers, such as temperature-sensitive PNIPAAm and $\mathrm{pH}$-sensitive chitosan, have also been used to formulate dual-responsive nanoparticles with combined properties of both the polymers [20]. As a result, these nanoparticles were shown to release the encapsulated drugs 
both at temperatures above the LCST and in an acidic environment, which are respectively beneficial attributes for cancer treatment since the tumor environment has higher temperature and lower $\mathrm{pH}(<7.2)$ compared to healthy tissues [21]. While polymer coatings play an important role in drug delivery and release kinetics, they also provide valuable functional groups for bioconjugation, which can be utilized to provide targeting moieties for MBTN.

In order to treat multi-drug resistant tumors, multiple drug loading strategies have been developed using magnetic liposomes. The bilayered geometry of liposomes allows for the encapsulation of multiple therapeutic agents for multidrug delivery [22]. For instance, hydrophilic drugs are incorporated in the hydrophilic core of the liposome, whereas hydrophobic drugs are loaded in the lipid bilayer of the liposome and amphiphilic molecules can be incorporated at the hydrophilic/hydrophobic interface of the liposome [23]. Amphiphilic poly $(2,2,3,4,4,4$-hexafluorobutyl methacrylate)g-PEG monomethacrylate (PHFMA-g-PEGMA) has also been used to prepare magnetic micelles of $\sim 100 \mathrm{~nm}$ diameter mainly for diagnosis of liver and spleen diseases. These nanoparticles showed high stability in water for up to 16 days and maintained sustained release of 5-fluorouracil for 40 hours. Further, these nanoparticles were successfully applied for in vivo MRI, which was evidenced by the dark contrast seen in the liver for up to 4 hours post-injection [24].

\section{TARGETING STRATEGIES}

A critical component in achieving an effective drug delivery and imaging tool is the ability to specifically target the diseased site and bypass healthy tissues. Targeting strategies for MBTN are met by various challenges such as selecting the appropriate target, methods to incorporate the correct targeting moieties, and strategies to avoid the rapid clearance of the delivery vehicles from the body [25]. The two basic mechanisms of targeting diseases are passive and active targeting, which is summarized in Fig. (2). Passive targeting is neither associated with the conjugation of antibodies nor influenced by any external forces. Instead, accumulation of the theranostic vehicle within the tumor site is accomplished by the enhanced permeability and retention (EPR effect) of tumor neovascularization [26]. The highly cluttered vasculature of the tumor tissue leads to a disorganized vasculature, and a defective lymphatic system [27]. When this occurs, nanoparticles in the range of 10 to $500 \mathrm{~nm}$ in diameter with hydrophilic surfaces have shown enhanced accumulation within the interstitial space of the tumor [28]. Hydrophilicity is an important factor as it not only increases the circulation time of nanoparticle, but also prevents nanoparticle from being cleared by macrophages and plasma protein adsorption [25]. Passive targeting was employed by Yu et al. [29] using doxorubicin-loaded thermally crosslinked MNPs for cancer treatment and imaging. The stable and protein-resistant coating of PEG-based poly(TMSMA-r-PEGMA) helped in increased nanoparticle circulation time and preferential accumulation in tumor region by the EPR effect in an orthotopic mouse model of lung cancer. Passive targeting can also be achieved through utilization of tumor activated prodrug therapy [25] or a polymer directed enzyme prodrug therapy (PDEPT) [30]. In PDEPT, polymeric prodrug containing linkers for cleavage are first delivered, which are in an inac- tive state during systemic circulation. Next, a polymer enzyme conjugate is delivered that activates the prodrug at the cancer site. PDEPT was successfully utilized by Fainaro et al. [30], where they experimented with non-mammalian enzymes for passively targeting tumors. To achieve this end, aHPMA conjugate containing enzyme, $\beta$-lactamase, was used to activate the HPMA-copolymer-methacryloylglycine-glycine-cephalosporin-doxorubicin prodrug component at the tumor site. Preliminary in vivo studies showed good cytocompatibility of the PDEPT combination and a significant decrease in tumor growth following administration when compared to the control group. Although nanoparticles can be used for drug delivery via passive targeting, this process can be both time-consuming and less effective due to accumulation in other healthy organs as well [31]. Therefore, alternative, more specific routes of targeting to the sites of interest are greatly needed.

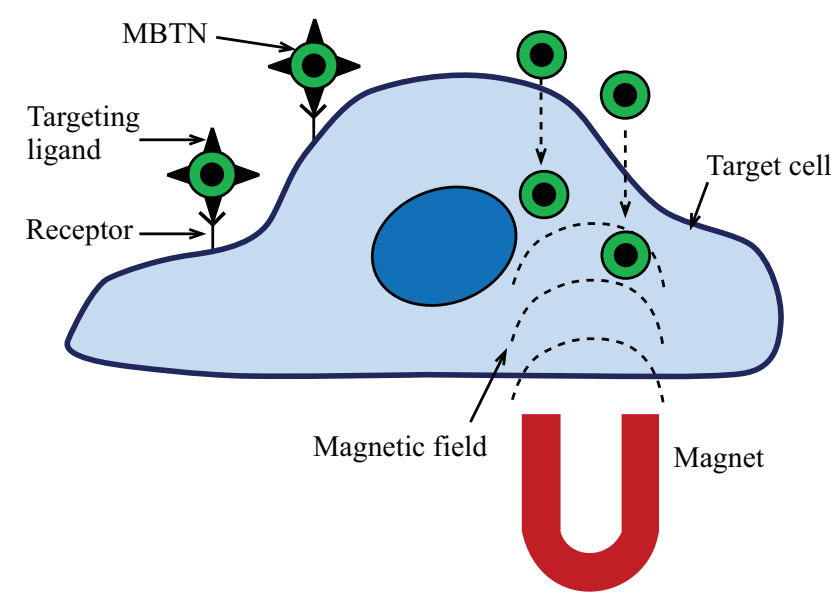

Fig. (2). Targeting mechanisms of MBTN emphasizing on receptormediated and magnetic targeting.

Unlike passive targeting, active targeting involves with either the conjugation of targeting ligands to nanoparticles or the use of external forces to guide the therapeutic vehicle to the diseased tissue [26]. A wide range of targeting moieties such as hormones, growth factors, proteins, peptides, and/or monoclonal and polyclonal antibodies have been used to direct MNPs to tumors. The choice of the targeting moiety is of great importance as it should be specific to the receptors over-expressed on the target cells. Such ligand-conjugated nanoparticles are engulfed by the receptor-mediated endocytosis process and destroyed intracellularly to release their therapeutic payload [32]. The receptor-mediated targeting is also useful for finding and destroying circulating or metastatic cells that express the receptors of interest [33]. Yao et al. [34] successfully conjugated A10 aptamers to thermally crosslinked MNPs to target prostate specific membrane antigens (PSMA) over-expressed by the prostate cancer cells. They observed that in media containing physiologic levels of folate, PSMA expression increased folic acid uptake approximately 2 -fold over non-expressing cells. Further, Wang et al. [35] have patented their theranostic nanoparticles for active targeting, diagnosis, and therapy of cancers. Poly(acrylic acid) (PAA) was coated on the MNP surface 
Table 1. Core, Shell, Imaging, Therapeutic, and Targeting Materials Used in MBTN Along with Application of MBTN

\begin{tabular}{|c|c|c|c|c|c|c|}
\hline Core & Shell & Imaging Agent & $\begin{array}{c}\text { Therapeutic } \\
\text { Agent }\end{array}$ & Targeting & Applications & Ref. \\
\hline SPION & PNIPAAm-AAm-AH & SPION & BSA & Magnetic & $\begin{array}{l}\text { Temperature-dependent drug } \\
\text { release }\end{array}$ & {$[12]$} \\
\hline SPION & $\begin{array}{l}\text { Chitosan-PNIPAAm- } \\
\text { N,N-DMAAm }\end{array}$ & SPION & Doxorubicin & Magnetic & $\begin{array}{l}\text { Temperature-dependent drug } \\
\text { release }\end{array}$ & {$[20]$} \\
\hline SPION & PEG & SPION & Doxorubicin & $\begin{array}{c}\text { Active } \\
\text { (folic acid) }\end{array}$ & $\begin{array}{l}\text { Anti-cancer drug delivery, } \\
\text { hyperthermia, MRI }\end{array}$ & {$[36]$} \\
\hline Ferro fluid & - & SPION & Mitoxanthrone & Magnetic & $\begin{array}{c}\text { Loco regional cancer treat- } \\
\text { ment }\end{array}$ & [39] \\
\hline $\begin{array}{l}\text { Manganese } \\
\text { oxide }\end{array}$ & PEI & Manganese oxide & siRNA & $\begin{array}{c}\text { Active } \\
\text { (Herceptin) }\end{array}$ & MRI, siRNA delivery & [42] \\
\hline $\begin{array}{l}\text { Gadolinium } \\
\text { oxide }\end{array}$ & PEG & Gadolinium oxide & Rhodamine & Passive & MRI & [43] \\
\hline SPION & PEI-QDs & SPION/QDs & & Magnetic & Dual-modality imaging & [48] \\
\hline SPION & PAA & $\begin{array}{l}\text { SPION/Di-alkylcarbocyanine } \\
\text { fluorescent dyes }\end{array}$ & Taxol & $\begin{array}{c}\text { Active } \\
\text { (folic acid) }\end{array}$ & MRI and optical imaging & [54] \\
\hline SPION & Glycol chitosan & SPION/Cy 5.5 & - & $\begin{array}{c}\text { Active } \\
\text { (Bombesin) }\end{array}$ & $\begin{array}{c}\text { Prostate cancer-specific deliv- } \\
\text { ery, imaging }\end{array}$ & {$[56]$} \\
\hline SPION & PEGylated chitosan & SPION & - & $\begin{array}{c}\text { Active } \\
\text { (Chlorotoxin) }\end{array}$ & Imaging, brain tumor targeting & {$[58]$} \\
\hline SPION & PCL & SPION & Gemcitabine & Magnetic & Anti-cancer drug delivery & {$[67]$} \\
\hline
\end{tabular}

and conjugated with pluronic F127 bound to folic acid, which is a targeting molecule. These nanoparticles were later loaded with Nile red and tested for their feasibility in vitro. The MNPs showed $\sim 80 \%$ cytocompatibility with KB oral epidermoid cells. Time-dependent uptake of the nanoparticles by folic acid receptor-expressing KB cells was seen by receptor-mediated endocytosis. Further, in vitro MRI studies showed greater negative contrast among KB cells incubated with folic acid-conjugated MNPs than with non-conjugated MNPs. Folic acid has also been used by Kaaki et al. [36] to conjugate with doxorubicin-loaded and PEG-coated MNPs for targeted breast cancer therapy via release of encapsulated doxorubicin. The folic acid-conjugated MNPs showed good stability and greater accumulation within MCF-7 breast cancer cells in vitro, when compared to non-conjugated MNPs. Moreover, Kievit et al. [37] have developed multifunctional MNPs tagged with HER2/neu antibody, which successfully bound to neu-expressing mammary carcinoma cells in mice. These MNPs could also specifically bind to metastatic cells in lung, liver, and bone marrow, thus demonstrating their potential in diagnosis and treatment of metastasized cancer.

In addition to receptor-mediated targeting, active targeting by the use of external forces like magnetic fields has been investigated. Magnetic targeting involves the delivery of MNPs locally, which can then be guided to the diseased site using an external magnetic field. MNPs become magnetized upon application of a magnetic field, and are quickly demagnetized when the magnetic field is removed due to superparamagnetic behavior [4]. Magnetic targeting is advantageous and more effective than passive targeting as rapid clearance of nanoparticles at specific disease sites by mononuclear macrophages can also be avoided [38]. Alexious et al. [39] recruited MNPs in the region of squamous cell carcinoma created in rabbits by using an external magnetic field of strength $1.7 \mathrm{~T}$. Another study conducted by Chertok et al. [40] imaged brain tumors non-invasively with MRI by concentrating MNPs at the tumor site by locally applied external magnetic field of about $4 \mathrm{~T}$. After effective targeting using MBTN, imaging of the delivery vehicles to highlight the diseased sites could be accomplished. The following section briefly describes the imaging agents used in the formulation of the MBTN.

\section{IMAGING AGENTS}

Imaging agents in theranostic nanomedicine play an important role in the diagnosis of a disease. Biodistribution, target accumulation, and pharmacokinetic activity of the nanomedicine for disease management can be visualized non-invasively and in real-time by the use of imaging agents [4]. The primary imaging agent used in the MBTN is SPION as it has been widely used as T2 negative contrast agents in MRI. Several SPION-based nanoparticles had been approved by the FDA for human use (Table 2) [41]. Examples include Feridex or ferumoxides as imaging agents for liver lesions; Combidex or ferumoxtran-10 for imaging of 'hidden' prostate cancer lymph node metastases; and Feraheme or feru- 
Table 2. FDA Approved MRI Contrast Agents

\begin{tabular}{|c|c|c|c|c|}
\hline Material & Commercial Name & Application & Status & Ref. \\
\hline SPION & Feridex IV/Ferumoxides & Liver MRI & Withdraw in 2008 & [41] \\
\hline SPION & Resovist/Cliavist & Liver MRI & Withdraw in 2009 & [41] \\
\hline SPION & Combidex/Ferumoxtran-10 & Prostate cancer lymph node metastases MRI & Withdraw in 2007 & {$[41]$} \\
\hline SPION & Feraheme/Ferumoxytol & Iron deficiency anemia treatment & Pending & [41] \\
\hline SPION & Lumirem/Gastromark & MRI of Gastrointestinal lumen & Approved in 1996 & [41] \\
\hline SPION & Clariscan/Feruglose & MR angiography, tumor microvasculature MRI & Development discontinued & {$[41]$} \\
\hline Gadolinium & Gadodiamide/Omniscan & Cranial and spinal MRI & Approved & {$[44]$} \\
\hline Gadolinium & Gadobenic acid/Multihance & MRI contrast agent & Approved & [44] \\
\hline Gadolinium & Gadopentetic acid/Magnevist & MRI of blood vessels and intracranial lesions & Approved & {$[44]$} \\
\hline Gadolinium & Gadoteridol/Prohance & MRI of central nervous system & Approved & {$[44]$} \\
\hline Gadolinium & Gadofosveset/Vasovist & MR angiography agent & Approved & {$[44]$} \\
\hline Gadolinium & Gadoversetamide/OptiMARK & Brain, spine, liver MRI & Approved & [44] \\
\hline Gadolinium & Gadoxetic acid/Eovist & Liver MRI & Approved & [44] \\
\hline
\end{tabular}

moxytol for treating iron deficiency anemia in chronic kidney diseases [10]. Further, manganese (Mn) and gadolinium (Gd)-based MRI contrast agents, such as multifunctional $\mathrm{MnO}$ and PEG functionalized $\mathrm{Gd}_{2} \mathrm{O}_{3}$ nanoparticles [42-44], have also been researched for in vitro and in vivo imaging applications, later was approved by FDA for human use. Gddiethylenetriamine pentaacetic acid (Gd-DTPA) complexes with anti-fibrin antibodies have been utilized for MRI, which proved the capability of these nanoparticles to enhance the MRI signal contrast over the clot surface [45]. Moreover, Gd-DTPA-bisoleate and Gd-DTPA-phosphatidyethanolamine were synthesized and their relaxivities were studied, and showed improved ion and particle relaxivity [46].

Contrast agents for optical imaging, PET or computed tomography (CT), have also been incorporated in MBTN to provide multi-modality imaging capabilities for enhanced and more accurate imaging of diseases and is summarized in Fig. (3). A multi-modality imaging approach has several advantages over a single modality system [47]. MRI provides exceptional tissue contrast, penetration depth, and high spatial resolution, whereas fluorescence imaging provides extremely high sensitivity and can be used for molecular imaging [25]. The most popular example of fluorescent agents used in optical imaging is metal semiconductor quantum dots (QD). QDs have remarkable optical properties compared to other fluorescent dyes [48]. QDs can emit light in the spectrum ranging from visible to near-infrared region, depending on their size or material composition like CdSe, $\mathrm{ZnS}$, and PbSe [25]. When QDs are used with MNPs, they are also called as magnetic QDs, which can be either heterodimers or homogeneous dispersion of QDs within MBTN [49]. Polyethyleneimine-capped QD were grafted on magnetite nanorings to develop magneto-fluorescent nanoprobes by Fan et al. [50]. In addition, Koole et al. [51] synthesized Gdbased lipid-coated silica nanoparticles with QD core as a new contrast agent platform for multimodality imaging. Moreover, fluorophores such as Alexa Fluor 647 have also been used along with MNPs for the applications in MRI and fluorescent imaging. Zhang et al. [52] patented their multifunctional nanoparticles for labeling and imaging of $\mathrm{T}$ cells.. They conjugated Alexa Fluor 647 and major histocompatibility complex (MHC) as a targeting molecule to the PEGylated iron oxide nanoparticles to specifically label T cells via MHC. Further, gold has also been used extensively along with MNPs for multifunctional imaging applications. Jiang et al. [53] developed bifunctional $\mathrm{Fe}_{3} \mathrm{O}_{4}-\mathrm{Ag}$ heterodimer nanoparticles of tunable sizes with high yield under mild conditions. The heterodimers also maintained high magnetic moments of $\mathrm{Fe}_{3} \mathrm{O}_{4}$. The macrophage cells labeled with these heterodimers were magnetically manipulated and imaged with two-photon fluorescence microscopy. Shi et al. [54] also anisotropically grew $\mathrm{PbSe}$ nanocrystals on $\mathrm{Au}-\mathrm{Fe}_{3} \mathrm{O}_{4}$ hybrid nanoparticles that are useful for magnetic-based targeting, delivery, cell separation, MRI, and fluorescencebased labeling applications.

PET isotopes such as ${ }^{18} \mathrm{~F}$ or ${ }^{64} \mathrm{Cu}$ and fluorescent dye VT680 have also been conjugated or encapsulated into the MBTN using azide-alkyne cycloaddition 'click' chemistry to enhance the sensitivity and reduce the dose required for clinical use [55]. Several other visible and near infrared fluorescent dyes, such as DiI/DiR, have been encapsulated in MBTN like poly(acrylic acid)-coated MNPs [56]. Moreover, bombesin was conjugated to dextran-coated MNPs and Nacetylhistidine-glycol chitosan nanoparticles for MRI application [57, 58]. Some other examples include rhodamine/ FITC-labeled paramagnetic nanoparticles [59] and Cy5.5labeled PEG/chitosan-coated MNPs [60]. However, currently used fluorescent tags are known to either be toxic or exhibit photobleaching. We have developed and patented a family of biodegradable photoluminescent polymers 


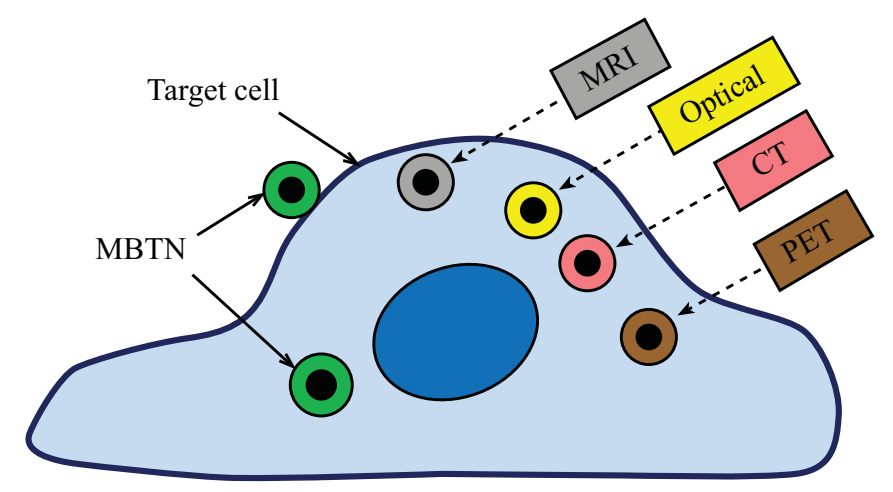

Fig. (3). Imaging modalities used for MBTN.
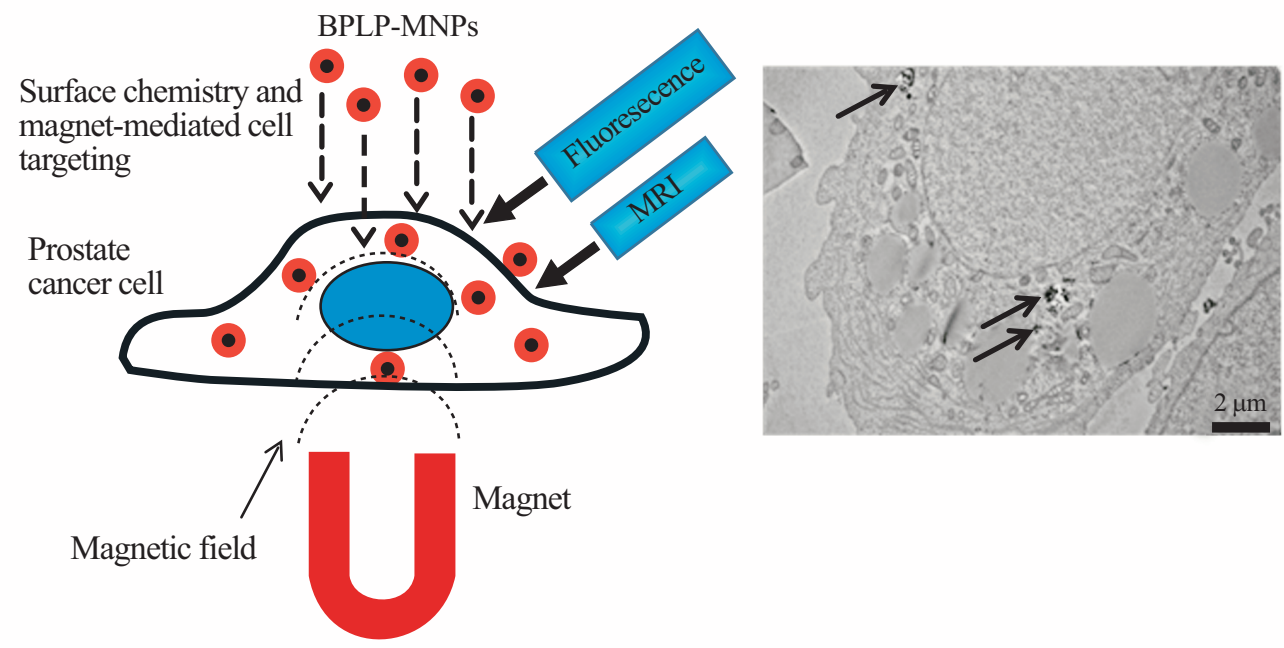

Fig. (4). Prostate cancer cell-specific dual-imaging enabled targeted BPLP-MNPs. Schematic representation of proposed working of system (left); and transmission electron microscopy image of cellular uptake of BPLP-MNPs (indicated by arrows) by prostate cancer cells (right).

(BPLPs) that are inherently fluorescent without conjugating any organic dyes or QDs [61, 62]. BPLPs are completely degradable, biocompatible, and display superior photoluminescent properties such as high quantum yield, photobleaching resistance, and tunable emission up to near infrared region when compared to currently used fluorescent tags [63]. We are currently developing BPLP-conjugated MNPs (BPLP-MNPs) for prostate cancer management as shown in Fig. (4). Our BPLP-MNPs provide dual-imaging (optical imaging and MRI) and dual-targeting (biomaterial-mediated targeting and magnetic targeting) capabilities. BPLP coating enables prostate cancer cell-selective uptake depending on its hydrophilicity level. We observed that hydrophilic BPLPMNPs were taken up more by metastatic PSMA- PC3 cells, whereas hydrophobic BPLP-MNPs were taken up more by non-metastatic PSMA $^{+} \mathrm{LNCaP}$ cells. The development of BPLP-MNPs may address the specific targeting, toxicity, and photobleaching concerns that arise when using organic dyes and cytotoxic QDs.

\section{THERAPEUTIC AGENTS}

Following disease diagnosis, a pivotal role of the MBTN is to treat the disease by either hyperthermia via alternating magnetic fields or releasing therapeutic agents as shown in Fig. (5). MBTN can be used without therapeutic agents to kill the heat-susceptible cancer cells by providing heat to the tumor region [5]. This is achieved by applying an external rotating or alternating magnetic field following the nanoparticle injection, which causes the MNPs to vibrate and generate heat to ultimately destroys the cancer cells [64]. In addition, MBTN have been used to deliver a wide variety of therapeutic agents ranging from chemotherapeutic drugs to peptides and genes $[7,8]$. Chemotherapeutic agents are more frequently loaded into the MBTN for the treatment of various tumors. For example, anticancer drug doxorubicin was loaded in liposomal nanoparticles containing dextran-coated MNPs as magneto-fluorescent agents for cancer chemotherapy [65]. Moreover, doxorubicin-loaded, oleic acidfunctionalized, and pluronic F-127-coated iron oxide nanoparticles were developed and patented by Labhasetwar et al. [66] for MRI and combinational anti-cancer therapies. These nanoparticles not only have a relatively higher T2 relaxivity compared to Feridex IV, but also contain the capability of accommodating high payloads of multiple drugs (95\% and $85 \%$ loading of paclitaxel and doxorubicin, respectively). The released drugs from these nanoparticles were effective in killing cancer cells and reducing tumor 
growth significantly. Further, novel biocompatible and biodegradable theranostic nanoparticles capable of targeted chemotherapeutic drug delivery, MRI, and optical imaging were also synthesized by Santa et al. [56]. In this study, PAA-coated MNPs were encapsulated with a near infrared dye and a chemotherapeutic drug (taxol) to serve the dual functions of diagnosis and treatment. Similarly, poly $(\varepsilon-$ caprolactone) (PCL)-coated MNPs encapsulating two anticancer drugs, Gemcitabine and Cisplatin, were prepared by Yang et al. [67], which could potentially be used for targeted drug delivery for cancer treatment. Other drugs used to date in the MBTN formulations include epirubicin [68] and mitoxantrone [69].

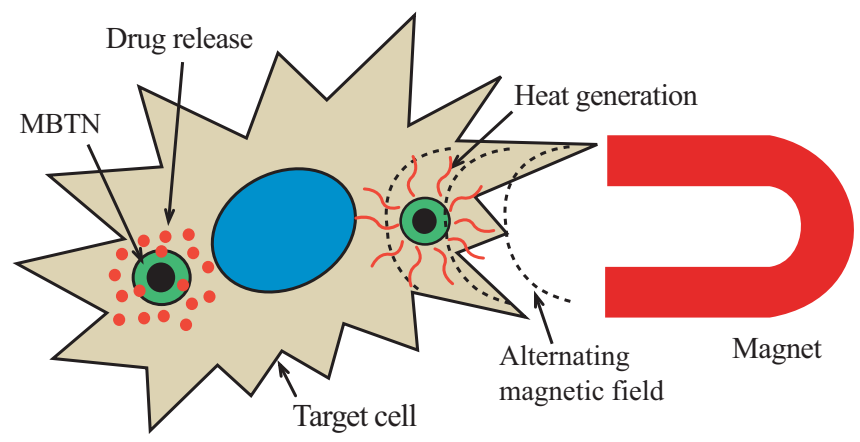

Fig. (5). Therapeutic action of MBTN showing drug release and hyperthermia treatment.

Besides chemotherapeutic reagents, MBTN have also been used to deliver bioactive molecules including doublestranded DNA (dsDNA), small interfering RNA (siRNA), and proteins. For instance, the MBTN containing both dsDNA and covalently bound doxorubicin molecules have been prepared for cancer treatment [70]. Recent research has also been focused on the development of polymer-coated iron oxide nanoparticles as agents for transfection and as DNA vaccine carriers [71]. Such nanoparticles can be used as effective DNA carriers for the transfection of cells and also as agents for vaccination. MNPs coated with polymers or proteins have shown success as transfection agents. Further, surface modified silica-iron oxide composite nanoparticles have also been used for delivering DNA to the targeted cells [71]. For gene delivery, stable lentiviral complexes were developed by Mykhaylyk et al. [72] using polyethylenimine-capped silica-iron oxide nanoparticles. Moreover, the MBTN have also been synthesized for small interfering RNA (siRNA) delivery. To achieve this end, Lee et al. [73] conjugated the siRNA onto the surface of the iron oxide nanoparticles along with the targeting moieties and fluorescent dyes for targeted siRNA delivery and fluorescence imaging. Another innovative example of siRNA delivery is the hollow manganese oxide nanoparticles prepared by Bae et al. [42] which was surface functionalized using 3,4-dihydroxyL-phenylalanine and incorporated therapeutic siRNA for simultaneous cancer diagnosis and treatment. In addition, protein delivery has also been implemented using MBTN. For example, Chertok et al. [74] synthesized $\beta$ Galactosidase-loaded heparin-coated MNPs for MRI and protein delivery to diagnose and treat brain tumor lesions.
The permeability to biological membranes imparted by the polyethylenimine-modified protein, together with magnetic targeting will help in selective accumulation of the nanoparticles at the tumor site.

\section{APPLICATIONS OF MBTN}

MNPs have been used in numerous applications, which can be categorized in three major application fields: (a) imaging (contrast agents for MRI), (b) therapy (chemotherapy via controlled drug release and hyperthermia via heat generation in alternating magnetic fields), and (c) cell separation (cell labeling/tracking and isolation using magnetic force). These are the mostly reported applications of MNPs, which have been covered in following sections.

\subsection{Imaging}

Nanotechnology has opened doors to new imaging agents that help not only in detection but also aid in management of diseases like cancer and cardiovascular diseases throughout the treatment. With imaging results, it is possible to determine if the treatment should be altered or terminated depending on the treatment efficacy or response of the disease [25]. Contrast agents serve as a powerful tool for characterization at the cellular and sub-cellular level. Due to the presence of MNPs, the MBTN are used as negative contrast agents in MRI. MNPs such as $\mathrm{Fe}_{3} \mathrm{O}_{4}, \mathrm{Gd}$, and $\mathrm{Mn}$ have high molar T2 relaxavities [75]. High spatial resolution provided by MRI and enhanced negative contrast provided by MNPs, when used with T2 weighted pulse sequences, make MRI attractive imaging modality for disease detection [28]. High spatial resolution of MRI and target specificity of MBTN allows the imaging of tumors as small as $2-3 \mathrm{~mm}$ in clinical applications [22]. Reddy et al. [76] tracked the migration of human bone derived mesenchymal stem cells in rabbit ischemic brain using chitosan-coated MNPs with MRI, which was also confirmed from several histological studies. In addition, Branca et al. [77] detected pulmonary micro-metastases with the help of luteinizing hormone-releasing, hormoneconjugated MNPs and MRI in mice bearing breast adenocarcinoma cells. Further, MBTN that have gained significant clinical attention, are iron oxide nanoparticles coated with either dextran or liposomes. Recent clinical trials have tried to image MNPs after administration in vivo. Clinical studies on prostate cancer patients by Harisinghani et al. [78] using lymphotropic MNPs, showed that the lymph node metastases could be accurately identified by high-resolution MR imaging of the MNPs. Phase I clinical trials on cancer patients using magnetic fluids bound to anticancer drugs such as epirubicin have shown that iron oxide could be guided to the region of interest using an external magnetic field and these fluids showed minimal toxicity in vivo [79].

MBTN have many times been used as dual-modality imaging agents. Optical imaging is often coupled with MRI, because optical imaging provides extremely high sensitivity and can be used for molecular imaging, thereby is widely used for in vivo applications [49]. A combination of MRI and fluorescence imaging was successfully achieved by Lee et al. [1] for the diagnosis of prostate cancer. Use of QDs and other fluorescent tags along with iron oxide has become a common multi-modal technique. Franck et al. [80] demon- 
strated the use of covalently attached fluorescent dye and MNPs in a nanoparticle formulation as a dual-imaging modality. Further, in another study, Li et al. [81] concluded that use of QDs was advantageous, as they exhibited bright fluorescence and did not undergo photobleaching as did the organic dyes.

\subsection{Chemotherapy}

With increasing prevalence rates of cancer, the management of cancer has become one of the leading research areas to find more effective imaging and therapeutic modalities. Traditional chemotherapies involve delivery of antineoplastic drugs to the cancer patients. Due to the non-specificity of these drugs, they manifest various side effects as a result of systemic toxicity [25]. The development of MBTN can also play a significant role in cancer management, due to their multi-functional capabilities. Using MBTN, systemic toxicity can be avoided by delivering the drugs only to the cancer cells via active/magnetic targeting while sparing healthy tissue and/or cells [25]. The dual-targeting mechanism (combination of receptor-mediated and magnetic targeting) may greatly reduce the toxicity of chemotherapeutic reagents by targeting cancer cells only and localizing of these drugs at the tumor site. Further, the chemotherapeutic drugs can be either loaded into the polymer shell or directly coated on the MNPs surface of MBTN. For example, Sun et al. [82] demonstrated extended particle retention and decreased survival rate in tumor cells when treated with methotrexate- and chlorotoxin-conjugated MNPs. Moreover, Yu et al. [29] developed doxorubicin-loaded thermally crosslinked MNPs that were administered intravenously into the tumor bearing mice to study the multi-functionality of the particles. The nanoparticles preferentially accumulated in the tumor region within 4.5 hours of the administration and were removed from the body within 24 hours. The nanoparticles showed their therapeutic effect within 12 hours of injection and a significant decrease in tumor size was noticed within 19 days of the treatment. Further, the MR imaging showed a strong negative contrast with the darkening of tumor region in the T2-weighted images, indicating accumulation of the nanoparticles. The results showed the potential of these theranostic MNPs for use in diagnosis (MRI) and treatment (drug release) of cancers.

\subsection{Hyperthermia}

Hyperthermia is a treatment in which high temperatures $\left(>41^{\circ} \mathrm{C}\right)$ are applied to kill cancer cells, as they are more sensitive to high temperatures unlike healthy cells [5]. The metallic and magnetic properties of MNPs make them suitable for hyperthermia treatment [83]. Upon administration and targeting of MBTN to cancer site, an alternating magnetic field can be applied, in which MNPs vibrate and generate thermal energy as a result of absorption of large amounts of magnetic energy by hysteresis loss [5]. Heat generated from the MNPs is affected by several factors, such as magnetic properties, particle size, amplitude and frequency of applied magnetic field, and cooling rate of blood [84]. Thus, by regulating these factors, the heat generation from MNPs can be controlled. However, an optimal hyperthermia effect can be achieved with $10 \mathrm{kA} / \mathrm{m}$ amplitude and $400 \mathrm{kHz}$ fre- quency [84]. The following is an example of the MBTN used for hyperthermia: Poloxamer, chitosan, alginate, and polyvinyl alcohol hydrogels loaded with MNPs were formulated by Renard et al. [85] and implanted in human cancer tumors xenografted in mice for hyperthermia treatment. In another study, Tseng et al. [86] proved that the viability of cancer cells significantly reduced when hyperthermia treatment was conducted using MNPs.

Hyperthermia has been used with other forms of therapy including chemotherapy to provide more effective treatment. At high temperatures, cancer cells become more vulnerable and respond to chemotherapeutic drugs or radiation effectively in an accelerated fashion [5]. Therefore, the combination of two therapies such as hyperthermia and chemotherapy or hyperthermia and radiation therapy would result in better treatment efficacies. For example, Wang et al. [87] synthesized MNPs encapsulated $\mathrm{As}_{2} \mathrm{O}_{3}$ nanoparticles for treating nude mice bearing xenograft human hepatocarcinoma with both thermal and chemo therapy. The application of two therapies in unison showed significant inhibitory effect over tumors in comparison to controls.

\subsection{Cell Separation/Isolation}

Applications of magnetism in cell separation have emerged in the last decade as an important driving force to separate magnetic from non-magnetic complexes in the cell mixtures. Magnetic cell separation permits isolation of specific cell types from crude samples such as blood, bone marrow, and cultivation media. The cell separation and purification process primarily consists of three steps: (a) incubating MBTN/MNPs coated with antibodies against interested cells with mixed cell suspension; (b) separating magnetic complex containing cells attached to MBTN/MNPs using an external magnetic force and washing it several times to remove contaminants; finally (c) either use the complexes directly or separate the cells from magnetic label, depending on the final application [88]. Various magnetic particles have been developed for use in separation processes including purification and immunoassays [75]. Cell separation with magnetic colloidal labels [89] and carbohydrate-coated (e.g., cellulose, sucrose) magnetic beads [90] have also been studied. In all cell isolation applications, MNPs are used to target and isolate a particular cell type using a ligand-receptor based mechanism leading to more specific cell isolation. For example, $\mathrm{Xu}$ et al. [91] isolated cancer cells from fresh whole blood using anti-HER2-conjugated amphiphilic polymercoated iron oxide nanoparticles. However, conventional MNPs do not support cell adhesion and cell growth on their surface. Another problem with the conventional MNP-based cell isolation system is that they are not loaded with proteins or growth factors for cell enrichment and differentiation.

\section{CURRENT \& FUTURE DEVELOPMENTS}

The primary goal in designing a MBTN is to create an advanced medical tool, which can detect and treat diseases in a single setting. Current developments of the MBTN are mainly focused on combining a therapeutic agent and an imaging agent to achieve dual-functionality. MBTN can be made more effective by incorporating additional functionalities to achieve multiple actions required for a disease man- 
agement in a single setting. Future developments of the MBTN will consist of active targeting capabilities and/or multi-modal imaging or therapeutic capabilities in conjunction with the current functionalities. The incorporation of multiple functionalities in a single system may raise several design consideration issues. There are several factors related to nanoparticles, which limit the development of an effective MBTN. Nanoparticle properties such as size, shape, surface charge, and surface modification are important factors to consider while designing MBTN with maximum effect at the target tissues while minimizing its clearance and toxicity to healthy tissues. Nanoparticle size plays a critical role in maintaining the magnetic properties and the rate of internalization by the target cells [92]. An optimum nanoparticle size between 10 to $100 \mathrm{~nm}$ prevents the removal of nanoparticles from circulation and enables them to pass through small capillaries. The size and shape of the particles can be manipulated by surfactant concentrations and types [93], while uniform sizes can be maintained by stirring the ferrofluid during preparation under constant temperature [94]. Further, a surface charge (zeta potential) of 10 to $30 \mathrm{mV}$ or -10 to $-30 \mathrm{mV}$ is optimum to achieve a stable nanoparticle suspension with minimal aggregation [95]. Polymer coatings and surface modifications can also affect the surface charge of the MBTN. For example, MNPs charged neutrally, positively and negatively were achieved by Villanueva et al. [96] using dextran, aminodextran, and heparin, respectively. The authors concluded that surface charge of the nanoparticles can play a major role in cellular uptake. The aminodextrancoated MNPs will be useful for hyperthermia due to their rapid accumulation within desired cells. The cationic charge on dextran-coated MNPs assists in uptake by HeLa cells following which live tracking of the cells can be carried out, where as heparin-coated MNPs proved to be toxic to the tested cells. Hydrophobic MNP surfaces can also be converted into hydrophilic by surface modification to prevent rapid clearance of particles from circulation. For instance, pullulan modified MNPs synthesized by Gupta et al. [92] resulted in increased hydrophilicity, reduced cytotoxicity, and better cellular uptake of particles.

Although MBTN have gained increased attention for biological and biomedical applications, it is critical to obtain more understanding on particle size control, in vivo particle degradation, distribution, and intracellular trafficking before it can move to clinical applications. Magnetic properties of MBTN are associated with the chemical composition, size, and morphology of the particles. For the efficient use of MBTN in targeting, imaging, and therapy, the particle size should be appropriate to allow attraction by magnetic field; the strength of magnetic field should be considerably strong in order to localize particles in the desired area; and finally particles may be injected in area accessible to tumor vasculature that would avoid reticulo-endothelial system. In vivo degradation of MBTN is mainly dependent on the dissociation of MNPs and the degradation of polymer coatings. Therefore, the polymer of choice should be highly biocompatible and biodegradable to avoid toxic effects to the other healthy organs. The chosen polymer should also provide functional groups for bioconjugation of targeting ligands to make MBTN site-specific. Further, understanding of the relaxivity of MBTN is highly essential for MRI and hyper- thermia applications. Relaxivity is dependent not only on magnetic properties but also on the applied magnetic field strength, temperature, and the medium in which the measurements are carried out. Equally as important, advances in imaging and diagnostic tools are also critical to realize the full potential of MBTN for disease management. Impressive developments in the nanotechnology and biomaterials fields have provided numerous tools and techniques to manipulate the nanoparticle properties. With the increasing rate of advances in nano-/bio-materials and the success of MNP-based nanoparticles in biomedical field, the clinical use of MBTN can be foreseen.

\section{CONFLICT OF INTEREST}

The authors confirm that this article content has no conflicts of interest.

\section{ACKNOWLEDGEMENTS}

We acknowledge the financial support from the Department of Defense (DOD,W81XWH-09-1-0313), the Cancer Prevention Research Institute of Texas (CPRIT,RP110412), the National Institute of Biomedical Imaging and Bioengineering (NIBIB,R21EB009795 and R01EB012575), and the National Science Foundation (NSF career award 0954109).

\section{REFERENCES}

[1] Xie J, Lee S, Chen X. Nanoparticle-based theranostic agents. Advanced Drug Delivery Reviews. 2010; 62(11): 1064-79.

[2] Bardhan R, Lal S, Joshi A, Halas NJ. Theranostic nanoshells: from probe design to imaging and treatment of cancer. Acc Chem Res. 2011 Oct 18 ; 44(10): 936-46.

[3] Babincova M, Babinec P. Magnetic drug delivery and targeting: principles and applications. Biomed Pap Med Fac Univ Palacky Olomouc Czech Repub. 2009 Dec; 153(4): 243-50.

[4] Bonnemain B. Superparamagnetic agents in magnetic resonance imaging: physicochemical characteristics and clinical applications. A review. Journal of drug targeting. 1998; 6(3): 167-74.

[5] Mornet S, Vasseur S, Grasset F, Duguet E. Magnetic nanoparticle design for medical diagnosis and therapy. Journal of Materials Chemistry. 2004; 14(14): 2161-75.

[6] Shubayev VI, Pisanic TR, $2^{\text {nd }}$, Jin S. Magnetic nanoparticles for theragnostics. Advanced drug delivery reviews. 2009 Jun 21; 61(6): 467-77.

[7] Strijkers GJ, Kluza E, Van Tilborg GA, van der Schaft DW, Griffioen AW, Mulder WJ, et al. Paramagnetic and fluorescent liposomes for target-specific imaging and therapy of tumor angiogenesis. Angiogenesis. 2010 Jun; 13(2): 161-73.

[8] Dobson J. Gene therapy progress and prospects: magnetic nanoparticle-based gene delivery. Gene therapy. 2006 Feb; 13(4): 283-7.

[9] Gupta AK, Wells S. Surface-modified superparamagnetic nanoparticles for drug delivery: preparation, characterization, and cytotoxicity studies. IEEE transactions on nanobioscience. 2004 Mar; 3(1): 66-73.

[10] Tassa C, Shaw SY, Weissleder R. Dextran-coated iron oxide nanoparticles: a versatile platform for targeted molecular imaging, molecular diagnostics, and therapy. Acc Chem Res. 2011 Oct 18; 44(10): 842-52.

[11] Arami H, Stephen Z, Veiseh O, Zhang M. Chitosan-coated iron oxide nanoparticles for molecular imaging and drug delivery. Advances in Polymer Science: Springer Berlin / Heidelberg 2011: 163-84.

[12] Rahimi M, Meletis EI, You S, Nguyen K. Formulation and characterization of novel temperature sensitive polymer-coated magnetic nanoparticles. J Nanosci Nanotechnol. 2010 Sep; 10(9): 6072-81.

[13] Tataru G, Popa M, Desbrieres J. Magnetic microparticles based on natural polymers. Int J Pharm. 2011 Feb 14; 404(1-2): 83-93.

[14] Tassa C, Shaw SY, Weissleder R. Dextran-Coated Iron Oxide Nanoparticles: A Versatile Platform for Targeted Molecular Imag- 
ing, Molecular Diagnostics, and Therapy. Accounts of Chemical Research. 2011 06/102012/05/01; 44(10): 842-52.

[15] Kaushik A, Khan R, Solanki PR, Pandey P, Alam J, Ahmad S, et al. Iron oxide nanoparticles-chitosan composite based glucose biosensor. Biosensors and Bioelectronics. 2008; 24(4): 676-83.

[16] Kim EH, Ahn Y, Lee HS. Biomedical applications of superparamagnetic iron oxide nanoparticles encapsulated within chitosan. Journal of Alloys and Compounds. 2007; 434â€"435(0): 633-6.

[17] Chattopadhyay P, Gupta RB. Supercritical CO2 based production of magnetically responsive micro- and nanoparticles for drug targeting. Ind Eng Chem Res. 2002 Oct 25; 41(24): 6049-58.

[18] Sahoo SK, Dilnawaz F, Singh A, inventors; Water dispersible glyceryl monooleate magnetic nanoparticle formulation. International patent WO2010/134087A1. 2010.

[19] Nguyen KT, Rahimi M, Kona S, Lin A, inventors; Board of Regents, The University of Texas System, Austin, TX, assignee. Compositions and methods for thermo-sensitive nanoparticles and magnetic nanoparticles. United Sates patent US2011/0097416A1. 2011.

[20] Yuan Q, Venkatasubramanian R, Hein S, Misra RDK. A stimulusresponsive magnetic nanoparticle drug carrier: Magnetite encapsulated by chitosan-grafted-copolymer. Acta Biomaterialia. 2008; 4(4): 1024-37.

[21] Fan L, Wu H, Zhang H, Li F, Yang T-h, Gu C-h, et al. Novel super $\mathrm{pH}$-sensitive nanoparticles responsive to tumor extracellular $\mathrm{pH}$ Carbohydrate Polymers. 2008; 73(3): 390-400.

[22] Kamaly N, Miller AD. Paramagnetic liposome nanoparticles for cellular and tumour imaging. Int J Mol Sci. 2010; 11(4): 1759-76.

[23] Namiki Y, Fuchigami T, Tada N, Kawamura R, Matsunuma S, Kitamoto $\mathrm{Y}$, et al. Nanomedicine for cancer: lipid-based nanostructures for drug delivery and monitoring. Acc Chem Res. 2011 Oct 18; 44(10): 1080-93.

[24] Li X, Li H, Liu G, Deng Z, Wu S, Li P, et al. Magnetite-loaded fluorine-containing polymeric micelles for magnetic resonance imaging and drug delivery. Biomaterials. 2012; 33(10): 3013-24

[25] Nie S, Xing Y, Kim GJ, Simons JW. Nanotechnology applications in cancer. Annual review of biomedical engineering. 2007; 9: 257-88.

[26] Danhier F, Feron O, Préat V. To exploit the tumor microenvironment: Passive and active tumor targeting of nanocarriers for anticancer drug delivery. J Control Release. 2010 Dec 1; 148(2): 135-46.

[27] Maeda H, Wu J, Sawa T, Matsumura Y, Hori K. Tumor vascular permeability and the EPR effect in macromolecular therapeutics: a review. Journal of Controlled Release. 2000; 65(1-2): 271-84.

[28] Sun C, Lee JS, Zhang M. Magnetic nanoparticles in MR imaging and drug delivery. Adv Drug Deliv Rev. 2008 Aug 17; 60(11): 1252-65.

[29] Yu MK, Jeong YY, Park J, Park S, Kim JW, Min JJ, et al. DrugLoaded Superparamagnetic Iron Oxide Nanoparticles for Combined Cancer Imaging and Therapy In Vivo. Angewandte Chemie International Edition. 2008; 47(29): 5362-5.

[30] Satchi-Fainaro R, Hailu H, Davies JW, Summerford C, Duncan R. PDEPT: polymer-directed enzyme prodrug therapy. 2. HPMA copolymer-beta-lactamase and HPMA copolymer-C-Dox as a model combination. Bioconjugate chemistry. 2003 Jul-Aug; 14(4): 797-804.

[31] Gao X, Cui Y, Levenson RM, Chung LWK, Nie S. In vivo cancer targeting and imaging with semiconductor quantum dots. Nat Biotech. $2004 ; 22(8)$ : 969-76.

[32] Cho K, Wang X, Nie S, Chen ZG, Shin DM. Therapeutic nanoparticles for drug delivery in cancer. Clin Cancer Res. 2008 Mar 1; 14(5): 1310-6.

[33] Marcucci F, Lefoulon F. Active targeting with particulate drug carriers in tumor therapy: fundamentals and recent progress. Drug Discovery Today. 2004; 9(5): 219-28.

[34] Yao V, Berkman CE, Choi JK, O'Keefe DS, Bacich DJ. Expression of prostate-specific membrane antigen (PSMA), increases cell folate uptake and proliferation and suggests a novel role for PSMA in the uptake of the non-polyglutamated folate, folic acid. Prostate. 2010 Feb 15; 70(3): 305-16.

[35] Wang LF, Lin JJ, Chen JS, Huang SJ, Ka JH, Wang YM, et al., inventors; Kaohsiung Medical University, Kaohsiung City, TW, assignee. Folic acid-mediated magnetic nanoparticle clusters for combined targeting, diagnosis, and therapy applications. United States patent US20110085987A1. 2011.

[36] Kaaki K, Herve-Aubert K, Chiper M, Shkilnyy A, Souce M, Benoit $\mathrm{R}$, et al. Magnetic nanocarriers of doxorubicin coated with poly(ethylene glycol) and folic acid: relation between coating struc- ture, surface properties, colloidal stability, and cancer cell targeting. Langmuir. 2011 Jan 17; 28(2): 1496-505.

[37] Kievit FM, Stephen ZR, Veiseh O, Arami H, Wang T, Lai VP, et al. Targeting of Primary Breast Cancers and Metastases in a Transgenic Mouse Model Using Rationally Designed Multifunctional SPIONs. ACS Nano. 2012 02/102012/04/27; 6(3): 2591-601.

[38] Blanco E, Kessinger CW, Sumer BD, Gao J. Multifunctional micellar nanomedicine for cancer therapy. Experimental biology and medicine (Maywood, NJ. 2009 Feb; 234(2): 123-31.

[39] Alexiou C, Arnold W, Klein RJ, Parak FG, Hulin P, Bergemann C, et al. Locoregional cancer treatment with magnetic drug targeting. Cancer research. 2000 Dec 1; 60(23): 6641-8.

[40] Chertok B, Moffat BA, David AE, Yu F, Bergemann C, Ross BD, et al. Iron oxide nanoparticles as a drug delivery vehicle for MRI monitored magnetic targeting of brain tumors. Biomaterials. 2008; 29(4): 487-96.

[41] Wang YX. Superparamagnetic iron oxide based MRI contrast agents: current status of clinical application. Quant Imaging Med Surg. 2011; 1(1): 35-40.

[42] Bae KH, Lee K, Kim C, Park TG. Surface functionalized hollow manganese oxide nanoparticles for cancer targeted siRNA delivery and magnetic resonance imaging. Biomaterials. 2011 Jan; 32(1): 176-84.

[43] Ahren M, Selegard L, Klasson A, Soderlind F, Abrikossova N, Skoglund C, et al. Synthesis and characterization of PEGylated $\mathrm{Gd} 2 \mathrm{O} 3$ nanoparticles for MRI contrast enhancement. Langmuir. 2010 Apr 20; 26(8): 5753-62.

[44] Geraldes CF, Laurent S. Classification and basic properties of contrast agents for magnetic resonance imaging. Contrast media \& molecular imaging. 2009 Jan-Feb; 4(1): 1-23.

[45] Vymazal J, Spuentrup E, Cardenas-Molina G, Wiethoff AJ, Hartmann MG, Caravan P, et al. Thrombus imaging with fibrin-specific gadolinium-based MR contrast agent EP-2104R: results of a phase II clinical study of feasibility. Invest Radiol. 2009 Nov; 44(11): 697-704.

[46] Winter PM, Caruthers SD, Yu X, Song SK, Chen J, Miller B, et al. Improved molecular imaging contrast agent for detection of human thrombus. Magn Reson Med. 2003 Aug; 50(2): 411-6.

[47] Schellenberger EA, Sosnovik D, Weissleder R, Josephson L. Magneto/optical annexin $\mathrm{V}$, a multimodal protein. Bioconjug Chem. 2004 Sep-Oct; 15(5): 1062-7.

[48] Medintz IL, Uyeda HT, Goldman ER, Mattoussi H. Quantum dot bioconjugates for imaging, labelling and sensing. Nature materials. 2005 Jun; 4(6): 435-46.

[49] Erathodiyil N, Ying JY. Functionalization of inorganic nanoparticles for bioimaging applications. Accounts of chemical research. 2011 Oct 18; 44(10): 925-35.

[50] Fan HM, Olivo M, Shuter B, Yi JB, Bhuvaneswari R, Tan HR, et al. Quantum dot capped magnetite nanorings as high performance nanoprobe for multiphoton fluorescence and magnetic resonance imaging. Journal of the American Chemical Society. 2010 Oct 27; 132(42): 14803-11.

[51] Koole R, van Schooneveld MM, Hilhorst J, Castermans K, Cormode DP, Strijkers GJ, et al. Paramagnetic lipid-coated silica nanoparticles with a fluorescent quantum dot core: a new contrast agent platform for multimodality imaging. Bioconjugate chemistry. 2008 Dec; 19(12): 2471-9.

[52] Zhang M, Gunn JW, Yee C, inventors; Fred Hutchinson Cancer Research Center and University of Washington, Seattle, WA, assignee. Magnetic nanoparticle and method for imaging T cells patent US2011/0250146A1. 2011.

[53] Jiang J, Gu H, Shao H, Devlin E, Papaefthymiou GC, Ying JY. Bifunctional Fe3O4-Ag heterodimer nanoparticles for two-photon fluorescence imaging and magnetic manipulation. Adv Mater. 2008; 20: 4403-7.

[54] Shi W, Sahoo Y, Zeng H, Ding Y, Swihart MT, Prasad PN. Anisotropic growth of $\mathrm{PbSe}$ nanocrystals on $\mathrm{Au}-\mathrm{Fe} 3 \mathrm{O} 4$ hybrid nanoparticles. Adv Mater. 2006; 18: 1889-94.

[55] Devaraj NK, Keliher EJ, Thurber GM, Nahrendorf M, Weissleder R. $18 \mathrm{~F}$ labeled nanoparticles for in vivo PET-CT imaging. Bioconjugate chemistry. 2009 Feb; 20(2): 397-401.

[56] Santra S, Kaittanis C, Grimm J, Perez JM. Drug/dye-loaded, multifunctional iron oxide nanoparticles for combined targeted cancer therapy and dual optical/magnetic resonance imaging. Small. 2009 Aug 17; 5(16): 1862-8.

[57] Martin AL, Hickey JL, Ablack AL, Lewis JD, Luyt LG, Gillies ER. Synthesis of bombesin-functionalized iron oxide nanoparticles and 
their specific uptake in prostate cancer cells. J Nanopart Res. 2010; 12(5): 1599-608

[58] Lee CM, Jeong HJ, Cheong SJ, Kim EM, Kim DW, Lim ST, et al. Prostate cancer-targeted imaging using magnetofluorescent polymeric nanoparticles functionalized with bombesin. Pharm Res. 2010 Apr; 27(4): 712-21.

[59] Schmieder AH, Caruthers SD, Zhang H, Williams TA, Robertson JD, Wickline SA, et al. Three-dimensional MR mapping of angiogenesis with alpha5betal(alpha nu beta3)-targeted theranostic nanoparticles in the MDA-MB-435 xenograft mouse model. FASEB J. 2008 Dec; 22(12): 4179-89.

[60] Veiseh O, Sun C, Fang C, Bhattarai N, Gunn J, Kievit F, et al. Specific targeting of brain tumors with an optical/magnetic resonance imaging nanoprobe across the blood-brain barrier. Cancer Res. 2009 Aug 1; 69(15): 6200-7.

[61] Yang J, Zhang Y, Gautam S, Liu L, Dey J, Chen W, et al. Development of aliphatic biodegradable photoluminescent polymers. Proc Natl Acad Sci U S A. 2009 Jun 23; 106(25): 10086-91.

[62] Yang J, Gautam S, inventors; Board of Regents, The University of Texas System, Austin, TX, assignee. Biodegradable photoluminescent polymers. United States patent US2011/0183435A1. 2011.

[63] Liu L, Zhang J, Su X, Mason RP. In vitro and in vivo assessment of $\mathrm{CdTe}$ and CdHgTe toxicity and clearance. Journal of biomedical nanotechnology. 2008 Dec 1; 4(4): 524-8.

[64] Dutz S, Kettering M, Hilger I, Muller R, Zeisberger M. Magnetic multicore nanoparticles for hyperthermia--influence of particle immobilization in tumour tissue on magnetic properties. Nanotechnology. $2011 \mathrm{Jul} 1 ; 22(26)$ : 265102.

[65] Erten A, Wrasidlo W, Scadeng M, Esener S, Hoffman RM, Bouvet $\mathrm{M}$, et al. Magnetic resonance and fluorescence imaging of doxorubicin-loaded nanoparticles using a novel in vivo model. Nanomedicine. 2010 Dec; 6(6): 797-807.

[66] Labhasetwar V, Leslie-Pelecky D, Jain T, inventors; University of Nebraska Medical Center, Omaha, NE, assignee. Multifunctional magnetic nanoparticles for imaging and drug delivery. United States patent US2007/0264199. 2007

[67] Yang J, Park SB, Yoon H-G, Huh YM, Haam S. Preparation of poly $\varepsilon$-caprolactone nanoparticles containing magnetite for magnetic drug carrier. International Journal of Pharmaceutics. 2006; 324(2): 185-90.

[68] McBain SC, Yiu HH, Dobson J. Magnetic nanoparticles for gene and drug delivery. Int J Nanomedicine. 2008; 3(2): 169-80.

[69] Tietze R, Schreiber E, Lyer S, Alexiou C. Mitoxantrone loaded superparamagnetic nanoparticles for drug targeting: a versatile and sensitive method for quantification of drug enrichment in rabbit tissues using HPLC-UV. J Biomed Biotechnol. 2010; 2010: 1-8.

[70] Nowicka AM, Kowalczyk A, Donten M, Krysinski P, Stojek Z. Influence of a magnetic nanoparticle as a drug carrier on the activity of anticancer drugs: Interactions of double stranded DNA and doxorubicin modified with a carrier. Analytical Chemistry. 2009 2012/02/03; 81(17): 7474-83.

[71] Xiang SD, Selomulya C, Ho J, Apostolopoulos V, Plebanski M. Delivery of DNA vaccines: an overview on the use of biodegradable polymeric and magnetic nanoparticles. Wiley Interdisciplinary Reviews: Nanomedicine and Nanobiotechnology. 2010; 2(3): 205-18.

[72] Mykhaylyk O, Sobisch T, Almstätter I, Sanchez-Antequera Y, Brandt S, Anton M, et al. Silica-iron oxide magnetic nanoparticles modified for gene delivery: A search for optimum and quantitative criteria. Pharm Res. 2012: 1-22.

[73] Lee JH, Lee K, Moon SH, Lee Y, Park TG, Cheon J. All-in-one target-cell-specific magnetic nanoparticles for simultaneous molecular imaging and siRNA delivery. Angew Chem Int Ed Engl. 2009; 48(23): 4174-9.

[74] Chertok B, David AE, Yang VC. Magnetically-enabled and MRmonitored selective brain tumor protein delivery in rats via magnetic nanocarriers. Biomaterials. 2011 Sept; 32(26): 6245-53.

[75] Ito A, Shinkai M, Honda H, Kobayashi T. Medical application of functionalized magnetic nanoparticles. Journal of bioscience and bioengineering. $2005 \mathrm{Jul}$; 100(1): 1-11.

[76] Reddy AM, Kwak BK, Shim HJ, Ahn C, Lee HS, Suh YJ, et al. In vivo tracking of mesenchymal stem cells labeled with a novel chi- tosan-coated superparamagnetic iron oxide nanoparticles using 3.0T MRI. Journal of Korean medical science. 2010 Feb; 25(2): 211-9.

[77] Branca RT, Cleveland ZI, Fubara B, Kumar CS, Maronpot RR, Leuschner C, et al. Molecular MRI for sensitive and specific detection of lung metastases. Proceedings of the National Academy of Sciences of the United States of America. 2010 Feb 23; 107(8): 3693-7.

[78] Harisinghani MG, Barentsz J, Hahn PF, Deserno WM, Tabatabaei $\mathrm{S}$, van de $\mathrm{Kaa} \mathrm{CH}$, et al. Noninvasive Detection of Clinically Occult Lymph-Node Metastases in Prostate Cancer. New England Journal of Medicine. 2003; 348(25): 2491-9.

[79] Lubbe AS, Bergemann C, Riess H, Schriever F, Reichardt P, Possinger $\mathrm{K}$, et al. Clinical experiences with magnetic drug targeting: a phase I study with 4 '-epidoxorubicin in 14 patients with advanced solid tumors. Cancer research. 1996 Oct 15; 56(20): 4686-93.

[80] Bertorelle F, Wilhelm C, Roger J, Gazeau F, Menager C, Cabuil V. Fluorescence-modified superparamagnetic nanoparticles: intracellular uptake and use in cellular imaging. Langmuir. 2006 Jun 6; 22(12): 5385-91.

[81] Li ZF, Ruckenstein E. Water-soluble poly(acrylic acid) grafted luminescent silicon nanoparticles and their use as fluorescent biological staining labels. Nano Letters. 2004 June 18; 4(8): 1463-7.

[82] Sun C, Fang C, Stephen Z, Veiseh O, Hansen S, Lee D, et al. Tumor-targeted drug delivery and MRI contrast enhancement by chlorotoxin-conjugated iron oxide nanoparticles. Nanomedicine (London, England). 2008 Aug; 3(4): 495-505.

[83] Berry CC, Curtis ASG. Functionalisation of magnetic nanoparticles for applications in biomedicine. J Phys D: Appl Phys. 2003; 36(13).

[84] Chen Y, Chen BA. Application and advancement of magnetic ironoxide nanoparticles in tumor-targeted therapy. Chinese journal of cancer. 2010 Jan; 29(1): 125-8.

[85] Le Renard PE, Jordan O, Faes A, Petri-Fink A, Hofmann H, Rufenacht $\mathrm{D}$, et al. The in vivo performance of magnetic particleloaded injectable, in situ gelling, carriers for the delivery of local hyperthermia. Biomaterials. 2010 Feb; 31(4): 691-705.

[86] Tseng HY, Lee GB, Lee CY, Shih YH, Lin XZ. Localised heating of tumours utilising injectable magnetic nanoparticles for hyperthermia cancer therapy. IET Nanobiotechnol. 2009 Jun; 3(2): 46-54.

[87] Wang ZY, Song J, Zhang DS. Nanosized As2O3/Fe2O3 complexes combined with magnetic fluid hyperthermia selectively target liver cancer cells. World J Gastroenterol. 2009 Jun 28; 15(24): 2995-3002.

[88] Safarík I, Safaríková M. Use of magnetic techniques for the isolation of cells. J Chromatogr B Biomed Sci Appl. 1999 Feb 5; 722 (1-2): 33-53.

[89] Owen CS. Magnetic cell sorting using collodial protein-magnetite. J Immunogenet. 1989 Apr; 16(2): 117-23.

[90] Rye PD. Sweet and sticky: carbohydrate-coated magnetic beads. Bio/Technology. 1996; 14: 155-7.

[91] Xu H, Aguilar ZP, Yang L, Kuang M, Duan H, Xiong Y, et al. Antibody conjugated magnetic iron oxide nanoparticles for cancer cell separation in fresh whole blood. Biomaterials. 2011 Dec; 32(36): 9758-65.

[92] Gupta AK, Gupta M. Cytotoxicity suppression and cellular uptake enhancement of surface modified magnetic nanoparticles. Biomaterials. 2005 May; 26(13): 1565-73

[93] Leem G, Sarangi S, Zhang S, Rusakova I, Brazdeikis A, Litvinov $\mathrm{D}$, et al. Surfactant-controlled size and shape evolution of magnetic nanoparticles. Cryst Growth Des. 2008 Dec 2; 9(1): 32-4.

[94] Rabias I, Tsitrouli D, Karakosta E, Kehagias T, Diamantopoulos G, Fardis $\mathrm{M}$, et al. Rapid magnetic heating treatment by highly charged maghemite nanoparticles on Wistar rats exocranial glioma tumors at microliter volume. Biomicrofluidics. 2010 June $21 ; 4(2)$

[95] He X, Wang K, Tan W, Liu B, Lin X, Huang S, et al. A novel gene carrier based on amino-modified silica nanoparticles. Chinese Science Bulletin. 2003; 48(3): 223-8.

[96] Villanueva A, nete MC, Roca AG, Calero M, VeintemillasVerdaguer S, Serna CJ, et al. The influence of surface functionalization on the enhanced internalization of magnetic nanoparticles in cancer cells. Nanotechnology. 2009 20(11): 115103. 